
Not for reproduction, distribution or commercial use.

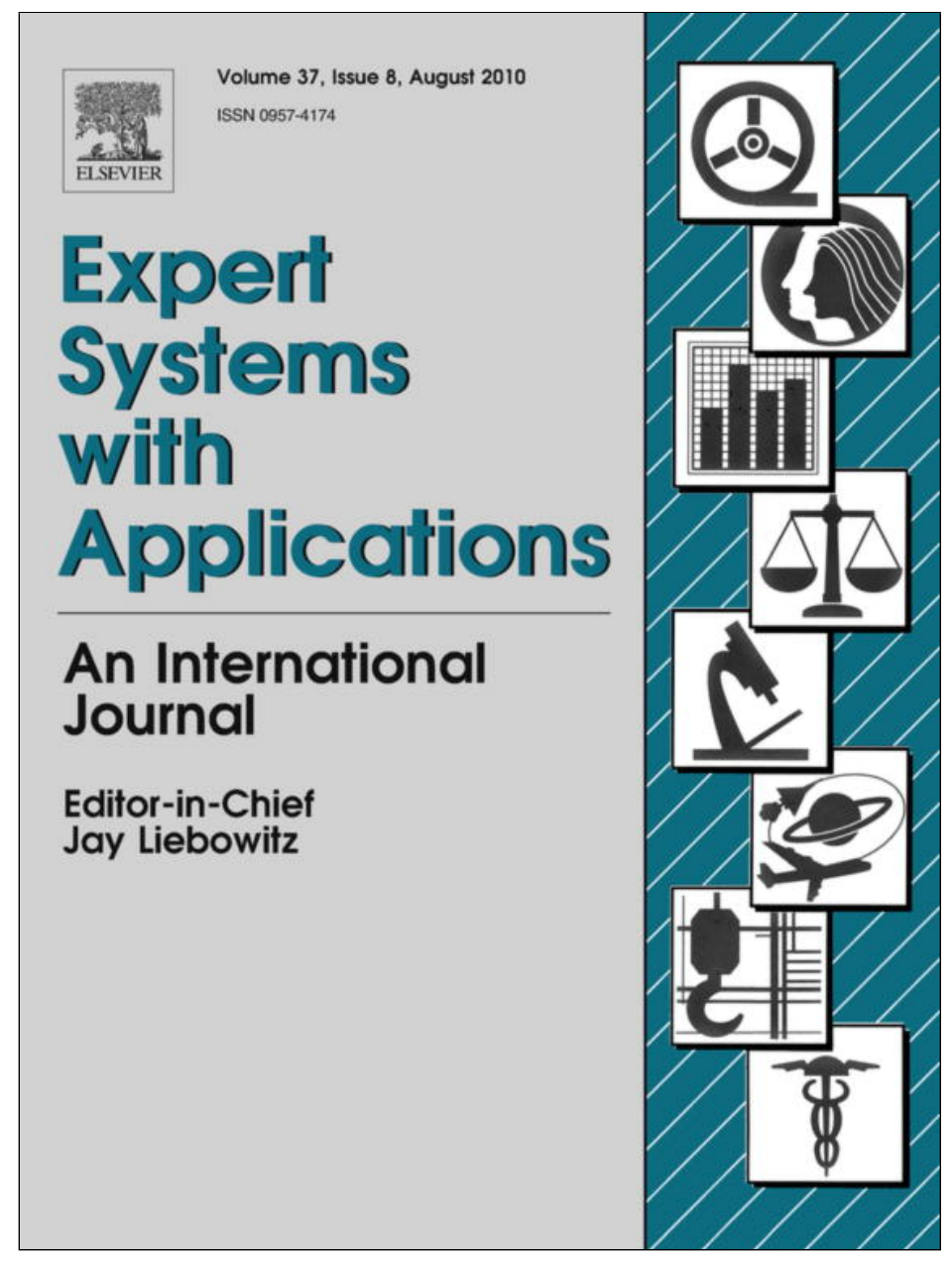

This article appeared in a journal published by Elsevier. The attached copy is furnished to the author for internal non-commercial research and education use, including for instruction at the authors institution and sharing with colleagues.

Other uses, including reproduction and distribution, or selling or licensing copies, or posting to personal, institutional or third party websites are prohibited.

In most cases authors are permitted to post their version of the article (e.g. in Word or Tex form) to their personal website or institutional repository. Authors requiring further information regarding Elsevier's archiving and manuscript policies are encouraged to visit: 


\title{
Bootstrap testing fuzzy hypotheses and observations on fuzzy statistic
}

\author{
Mohammad Ghasem Akbari ${ }^{\mathrm{a}, *}$, Abdolhamid Rezaei ${ }^{\mathrm{b}}$ \\ ${ }^{a}$ Department of Statistics, Faculty of Sciences, University of Birjand, South Khorasan, Iran \\ ${ }^{\mathrm{b}}$ Department of Statistics, School of Mathematical Sciences, Ferdowsi University of Mashhad, Mashad, Iran
}

\section{A R T I C L E I N F O}

\section{Keywords:}

Fuzzy canonical number

Yao-Wu signed distance

Fuzzy data

Fuzzy hypotheses

Fuzzy confidence

Testing hypotheses

Bootstrap method

\begin{abstract}
A B S T R A C T
The bootstrap is a simple and straightforward method for calculating approximated biases, standard deviations, confidence intervals, testing statistical hypotheses, and so forth, in almost any nonparametric estimation problem. A new approach for bootstrap testing fuzzy hypotheses based on fuzzy test statistic is introduced. In this paper we describe bootstrap method that is designed directly for hypothesis testing for fuzzy data based on Yao-Wu signed distance.
\end{abstract}

(c) 2010 Elsevier Ltd. All rights reserved.

\section{Introduction}

Statistical analysis, in traditional form, is based on crispness of data, random variable, point estimation, hypotheses, parameter and so on. As there are many different situations in which the above mentioned concepts are imprecise. The point estimation approaches frequently are used in statistical inference. On the other hand, the theory of fuzzy sets is a well known tool for formulation and analysis of imprecise and subjective concepts. Therefore the testing hypotheses with fuzzy data can be important. The problem of testing statistical hypotheses when the hypotheses are crisp or fuzzy have been studied by a few authors.

Arnold $(1996,1998)$ presented an approach to test fuzzily formulated hypotheses, in which he considered fuzzy constraints on the type $I$ and II errors. Taheri and Behboodian (1991) state and prove a Neyman-Pearson Lemma for testing fuzzy hypotheses. Their approach has been extended by Torabi, Behboodian, and Taheri (2006) for the cases when the data are fuzzy, too. For some other recent works in testing hypothesis using fuzzy approaches, see Buckley (2005, 2006), Desimpelaere and Marchant (2007), Filzmoser and Viertl (2004), Hyniewicz (2006), Thompson and Geyer (2007), Viertl (2006).

The bootstrap using fuzzy data, is developed in different approaches.

Montenegro, Colubi, Casals, and Gil (2004) have presented asymptotic one-sample procedure. Korner's asymptotic development (2000) concerns general fuzzy random variables (taking on way-either finite or infinite-number of values in the space of compact convex fuzzy sets of a finite-dimensional Euclidean space). Gonzalez-Rodriguez, Montenegro, Colubi, and Gil (2006) have

\footnotetext{
* Corresponding author. Tel./fax: +98 5118828605 .

E-mail address: g_z_akbari@yahoo.com (M.G. Akbari).
}

shown that the one-sample method of testing the mean of a fuzzy random variable can be extended to general ones (more precisely, to those whose range is not necessarily finite and whose values are fuzzy subsets of finite-dimensional Euclidean space).

In this paper we construct a new method for bootstrap testing hypotheses in fuzzy environment which is completely different from those mentioned above. For this purpose we organize the matter in the following way:

in Section 2 we describe some basic concepts of canonical fuzzy numbers, Yao and $\mathrm{Wu}(2000)$ signed distance and fuzzy hypotheses. In Section 3 we come up bootstrap testing fuzzy hypotheses based on fuzzy test statistic for mean based on Yao-Wu signed distance. Section 4 provide a Bootstrap testing fuzzy hypotheses based on fuzzy test statistic for variance based on Yao-Wu signed distance. A brief conclusion is provided in Section 5 .

\section{Preliminaries}

In this section we study canonical fuzzy numbers, Yao-Wu signed distance and fuzzy hypotheses.

\subsection{Canonical fuzzy numbers}

Let $X$ be the universal space, then a fuzzy subset $\tilde{x}$ of $X$ is defined by its membership function $\mu_{\tilde{x}}: X \rightarrow[0,1]$. We denote by $\tilde{x}_{\alpha}=$ $\left\{x: \mu_{\tilde{x}}(x) \geqslant \alpha\right\}$ the $\alpha$-cut set of $\tilde{x}$ and $\tilde{x}_{0}$ is the closure of the set $\left\{x: \mu_{\tilde{x}}(x)>0\right\}$, and

(1) $\tilde{x}$ is called normal fuzzy set if there exist $x \in X$ such that $\mu_{\tilde{x}}(x)=1$

(2) $\tilde{x}$ is called convex fuzzy set if $\mu_{\tilde{x}}(\lambda x+(1-\lambda) y) \geqslant \min$ $\left(\mu_{\tilde{x}}(x), \mu_{\tilde{x}}(y)\right)$ for all $\lambda \in[0,1]$; 
(3) the fuzzy set $\tilde{x}$ is called a fuzzy number if $\tilde{x}$ is normal convex fuzzy set and its $\alpha$-cut sets are bounded $\forall \alpha \neq 0$;

(4) $\tilde{x}$ is called a closed fuzzy number if $\tilde{x}$ is fuzzy number and its membership function $\mu_{\tilde{x}}$ is upper semicontinuous;

(5) $\tilde{x}$ is called a bounded fuzzy number if $\tilde{x}$ is a fuzzy number and its membership function $\mu_{\tilde{x}}$ has compact support.

If $\tilde{x}$ is a closed and bounded fuzzy number with $x_{\alpha}^{L}=\inf \left\{x: x \in \tilde{x}_{\alpha}\right\}$ and $x_{\alpha}^{U}=\sup \left\{x: x \in \tilde{x}_{\alpha}\right\}$ and its membership function is strictly increasing on the interval $\left[x_{\alpha}^{L}, x_{1}^{L}\right]$ and strictly decreasing on the interval $\left[x_{1}^{U}, x_{\alpha}^{U}\right]$ for any $\alpha \in[0,1]$, then $\tilde{x}$ is called canonical fuzzy number.

Let " $\odot$ " be a binary operation $\oplus$ or $\ominus$ between two canonical fuzzy numbers $\tilde{a}$ and $\tilde{b}$. The membership function of $\tilde{a} \odot \tilde{b}$ is defined by

$\mu_{\tilde{a} \odot \tilde{b}}(z)=\sup _{x \circ y=z} \min \left\{\mu_{\tilde{a}}(x), \mu_{\tilde{b}}(y)\right\} \quad \forall z \in \mathcal{R}$

for $\odot=\oplus$ or $\ominus$ and $\circ=+$ or - .

In the following, let $\odot_{i n t}$ be a binary operation $\oplus_{\text {int }}$ or $\ominus_{\text {int }}$ between two closed intervals $\tilde{a}_{\alpha}=\left[a_{\alpha}^{L}, a_{\alpha}^{U}\right]$ and $\tilde{b}_{\alpha}=\left[b_{\alpha}^{L}, b_{\alpha}^{U}\right]$. Then $\tilde{a}_{\alpha} \odot_{i n t} \tilde{b}_{\alpha}$ is defined by

$\tilde{a}_{\alpha} \odot_{i n t} \tilde{b}_{\alpha}=\left\{z \in \mathcal{R}: z=x \circ y, x \in \tilde{a}_{\alpha}, y \in \tilde{b}_{\alpha}\right\}$.

If $\tilde{a}$ and $\tilde{b}$ be two closed fuzzy numbers. Then $\tilde{a} \oplus \tilde{b}$ and $\tilde{a} \ominus \tilde{b}$ are also closed fuzzy numbers. Furthermore, we have

$$
\begin{aligned}
& (\tilde{a} \oplus \tilde{b})_{\alpha}=\tilde{a}_{\alpha} \oplus_{i n t} \tilde{b}_{\alpha}=\left[a_{\alpha}^{L}+b_{\alpha}^{L}, a_{\alpha}^{U}+b_{\alpha}^{U}\right] \\
& (\tilde{a} \ominus \tilde{b})_{\alpha}=\tilde{a}_{\alpha} \ominus_{i n t} \tilde{b}_{\alpha}=\left[a_{\alpha}^{L}-b_{\alpha}^{U}, a_{\alpha}^{U}-b_{\alpha}^{L}\right]
\end{aligned}
$$

\subsection{Yao-Wu signed distance}

Now we define a signed distance between fuzzy numbers which is used later.

Several ranking methods have been proposed so far, by Cheng (1998), Modarres and Sadi-Nezhad (2001) and Nojavan and Ghazanfari (2006). In this paper we use another ranking system for canonical fuzzy numbers which is very realistic and is defined by Yao and $\mathrm{Wu}$ as the following:

Definition 2.1. For each $a, b \in \mathcal{R}$, define the signed distance $d^{*}$ of $a$ and $b$ by $d^{*}(a, b)=a-b$. Thus, we have the following way to define the rank of any two numbers on $\mathcal{R}$. For each $a, b \in \mathcal{R}$,

$$
\begin{aligned}
& d^{*}(a, b)>0 \Longleftrightarrow d^{*}(a, 0)>d^{*}(b, 0) \Longleftrightarrow a>b, \\
& d^{*}(a, b)<0 \Longleftrightarrow d^{*}(a, 0)<d^{*}(b, 0) \Longleftrightarrow a<b, \\
& d^{*}(a, b)=0 \Longleftrightarrow d^{*}(a, 0)=d^{*}(b, 0) \Longleftrightarrow a=b .
\end{aligned}
$$

Definition 2.2. For each $\tilde{a}, \tilde{b} \in \mathcal{F}(\mathcal{R})$, define the signed distance of $\tilde{a}$ and $\tilde{b}$ as follows:

$\mathrm{d}(\tilde{a}, \tilde{b})=\int_{0}^{1}\left(M_{\alpha}(\tilde{a})-M_{\alpha}(\tilde{b})\right) \mathrm{d} \alpha=\int_{0}^{1} d^{*}\left(M_{\alpha}(\tilde{a}), M_{\alpha}(\tilde{b})\right) \mathrm{d} \alpha$,

where $M_{\alpha}(\tilde{a})$ and $M_{\alpha}(\tilde{b})$ are equal to $\frac{a_{\alpha}^{L}+a_{\alpha}^{U}}{2}$ and $\frac{b_{\alpha}^{L}+b_{\alpha}^{U}}{2}$, respectively, furthermore $\mathrm{d}(\tilde{a}, \tilde{b})$ means the distance of $\tilde{a}$ to $\tilde{b}$.

Definition 2.3 (Yao and $W u, 2000$ ). For each $\tilde{a}, \tilde{b} \in \mathcal{F}(\mathcal{R})$, define the ranking $\prec, \succ$ and $\approx$ of $\tilde{a}$ and $\tilde{b}$ by

$$
\begin{aligned}
& \mathrm{d}(\tilde{a}, \tilde{b})>0 \Longleftrightarrow \mathrm{d}(\tilde{a}, 0)>\mathrm{d}(\tilde{b}, 0) \Longleftrightarrow \tilde{a} \succ \tilde{b}, \\
& \mathrm{~d}(\tilde{a}, \tilde{b})<0 \Longleftrightarrow \mathrm{d}(\tilde{a}, 0)<\mathrm{d}(\tilde{b}, 0) \Longleftrightarrow \tilde{a} \prec \tilde{b}, \\
& \mathrm{~d}(\tilde{a}, \tilde{b})=0 \Longleftrightarrow \mathrm{d}(\tilde{a}, 0)=\mathrm{d}(\tilde{b}, 0) \Longleftrightarrow \tilde{a} \approx \tilde{b} .
\end{aligned}
$$

\subsection{Fuzzy hypotheses}

We define some models, as fuzzy sets of real numbers, for modeling the extended versions of the simple, the onesided, and the two-sided ordinary (crisp) hypotheses to the fuzzy ones.

Testing statistical hypothesis is a main branch of statistical inference. Typically, a statistical hypothesis is an assertion about the probability distribution of one or more random variable(s). Traditionally, all statisticians assume the hypothesis for which we wish to provide a test are well-defined. This limitation, sometimes, forces the statistician to make decision procedure in an unrealistic manner. This is because in realistic problems, we may come across non-precise (fuzzy) hypothesis. For example, suppose that $\theta$ is the proportion of a population which has a disease. We take a random sample of elements and study the sample for having some idea about $\theta$. In crisp hypothesis testing, one uses the hypotheses of the form: $H_{0}: \theta=0.2$ versus $H_{1}: \theta \neq 0.2$ or $H_{0}: \theta \leqslant 0.2$ versus $H_{0}: \theta>0.2$, and so on. However, we would sometimes like to test more realistic hypotheses. In this example, more realistic expressions about $\theta$ would be considered as: small, very small, large, approximately 0.2 , and so on. Therefore, more realistic formulation of the hypotheses might be $H_{0}: \theta$ is small, versus $H_{1}: \theta$ is not small. We call such expressions as fuzzy hypotheses.

We define some models, as fuzzy sets of real numbers, for modeling the extended versions of the simple, the one-sided, and the two-sided crisp hypotheses to the fuzzy ones.

Definition 2.4. Let $\theta_{0}$ be a real number and known.

(i) Any hypothesis of the form $\left(H: \theta\right.$ is approximately $\left.\theta_{0}\right)$ is called to be a fuzzy simple hypothesis.

(ii) Any hypothesis of the form $\left(H: \theta\right.$ is not approximately $\left.\theta_{0}\right)$ is called to be a fuzzy two-sided hypothesis.

(iii) Any hypothesis of the form ( $H: \theta$ is essentially smaller than $\left.\theta_{0}\right)$ is called to be a fuzzy left one-sided hypothesis.

(iv) Any hypothesis of the form $\left(H_{1}: \theta\right.$ is essentially larger than $\left.\theta_{0}\right)$ is called to be a fuzzy right one-sided hypothesis.

We denote the above definitions by

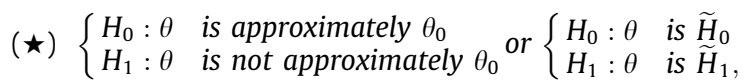

$(\star \star)\left\{\begin{array}{ll}H_{0}: \theta & \text { is approximately } \theta_{0} \\ H_{1}: \theta & \text { is certainly larger than } \theta_{0}\end{array}\right.$ or $\begin{cases}H_{0}: \theta & \text { is } \widetilde{H}_{0 L} \\ H_{1}: \theta & \text { is } \widetilde{H}_{1},\end{cases}$

$(\star \star \star)\left\{\begin{array}{ll}H_{0}: \theta & \text { is approximately } \theta_{0} \\ H_{1}: \theta & \text { is certainly smaller than } \theta_{0}\end{array}\right.$ or $\begin{cases}H_{0}: \theta & \text { is } \widetilde{H}_{0 R} \\ H_{1}: \theta & \text { is } \widetilde{H}_{1}\end{cases}$

The above areas are shown in Figs. 1-3.

\section{Bootstrap testing fuzzy hypotheses based on fuzzy test statistic for mean}

Suppose that we have canonical fuzzy random sample $\tilde{\mathbf{x}}=\left(\tilde{x}_{1}, \tilde{x}_{2}, \ldots, \tilde{x}_{n}\right)$.

3.1. Testing fuzzy simple hypothesis fuzzy against the fuzzy two-sided hypothesis

We want to test the fuzzy null hypotheses

$\widetilde{H}_{0}$ : the mean of observations $(\theta)$ is approximately $\theta_{0}$ versus $\widetilde{H}_{1}$ : the mean of observations $(\theta)$ is not approximately $\theta_{0}$. 


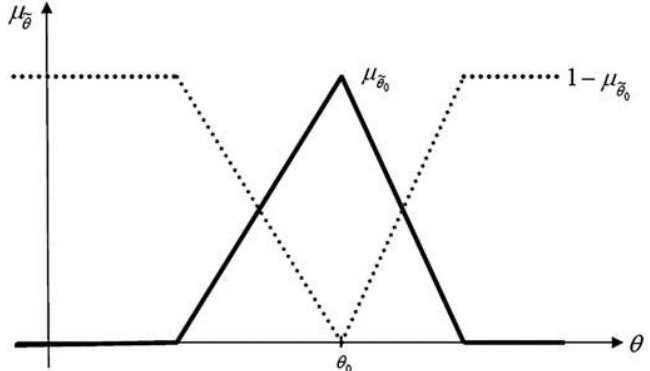

Fig. 1. The fuzzy hypotheses $\widetilde{H}_{0}$ versus $\widetilde{H}_{1}$

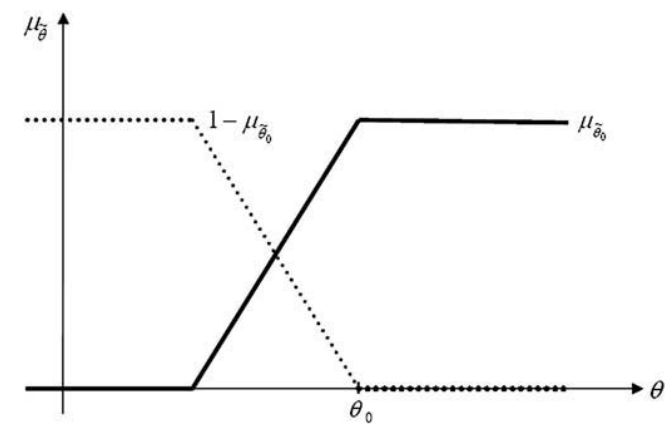

Fig. 2. The fuzzy hypotheses $\widetilde{H}_{0 L}$ versus $\widetilde{H}_{1}$

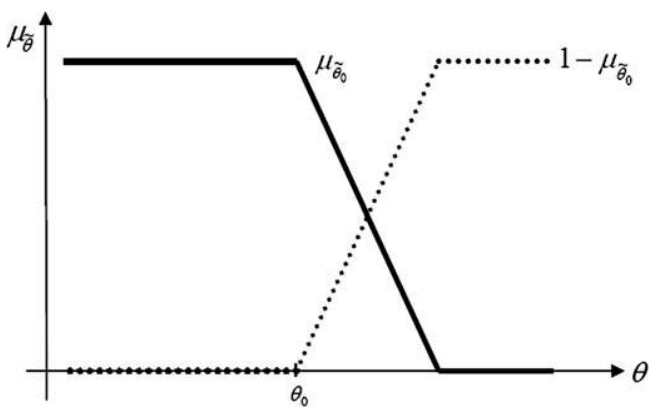

Fig. 3. The fuzzy hypotheses $\widetilde{H}_{0 R}$ versus $\widetilde{H}_{1}$.

We generate $B$ bootstrap fuzzy random sample $\tilde{\mathbf{x}}^{*^{1}}, \tilde{\mathbf{x}}^{*^{2}}, \ldots, \tilde{\mathbf{x}}^{*^{B}}$ (i.e., each $\tilde{\mathbf{x}}^{{ }^{b}}$ is a fuzzy sample of size $n$ drawn randomly and replacement from $\tilde{\mathbf{x}}$.

We need a distribution that estimates the population of treatment times under $H_{0}$. Note first that the empirical distribution (i.e., putting probability $\frac{1}{n}$ on each member of $\tilde{\mathbf{x}}$ ) is not an approximate estimate for distribution because it does not obey $\mathrm{H}_{0}$. Somehow we need to obtain an estimate of the population that has mean $\tilde{\theta_{0}}$. A simple way is to translate the empirical distribution so that it has the desired mean. In other words, we use as our estimated null distribution the empirical distribution on the values

$\tilde{x}_{c i}=\tilde{x}_{i} \ominus \widetilde{\overline{\mathbf{x}}} \oplus \widetilde{\theta_{0}}, \quad i=1,2, \ldots, n$,

because $\frac{1}{n} \oplus_{i=1}^{n} \tilde{x}_{c i}=\widetilde{\theta_{0}}$.

We compute

$t\left(\tilde{\mathbf{x}}_{c}^{*^{b}}\right)=\frac{\mathrm{d}\left(\widetilde{\mathbf{x}}_{c}^{*^{b}}, \widetilde{\theta}_{0}\right)}{\widehat{\operatorname{Se}}^{*^{b}}\left(\widetilde{\mathbf{X}}_{c}\right)} \quad b=1,2, \ldots, B$, where

(1) $d$ is Yao-Wu signed distance.

(2) $\widetilde{\mathbf{x}}_{c}^{*^{b}}=\frac{1}{n} \oplus_{i=1}^{n} \tilde{x}_{c i}^{* b} b=1,2, \ldots, B$.

(3) $\widehat{S e}^{*^{b}}\left(\widetilde{\mathbf{x}}_{c}\right)=\sqrt{\frac{1}{n(n-1)} \sum_{i=1}^{n} d^{2}\left(\tilde{x}_{c i}^{* b}, \widetilde{\mathbf{x}}_{c}^{* b}\right)} b=1,2, \ldots, B$.

The $\gamma$ th percentile of $t\left(\tilde{\mathbf{x}}_{c}^{*^{b}}\right)$ is estimated by the value $\hat{t}^{\gamma}$ such that $\frac{\#\left\{t\left(\tilde{\mathbf{x}}_{c}^{b}\right) \leqslant \hat{t}^{\gamma}\right\}}{B}=\gamma$.

Finally, the fuzzy bootstrap confidence interval at the level $1-2 \gamma$ using fuzzy data is

$$
\begin{aligned}
\widetilde{\Pi}_{\alpha}=\left\{\left[\frac{1}{n} \sum_{i=1}^{n} x_{i}-\hat{t}^{\gamma} \frac{s_{(\tilde{\mathbf{x}})}}{\sqrt{n}}, \quad \frac{1}{n} \sum_{i=1}^{n} x_{i}-\hat{t}^{1-\gamma} \frac{s_{(\tilde{\mathbf{x}})}}{\sqrt{n}}\right],\right. \\
\left.x_{i} \in \tilde{x}_{i \alpha}, i=1,2, \ldots, n\right\},
\end{aligned}
$$

where $s_{(\tilde{\mathbf{x}})}^{2}=\frac{1}{n-1} \sum_{i=1}^{n} d^{2}\left(\tilde{x}_{i}, \tilde{\mathbf{x}}\right)$.

If $B \times \gamma$ is not an integer, the following procedure can be used. Assuming $\gamma \leqslant 0.5$, let $k=[(B+1) \gamma]$, the largest integer $\leqslant(B+1) \gamma$. Then we define the empirical $\gamma$ and $1-\gamma$ quantizes by the $k$ th largest and $(B+1-k)$ th largest values of $t\left(\tilde{\mathbf{x}}_{c}^{*^{b}}\right)$, respectively.

Example 3.1. Suppose that we have taken a fuzzy random sample of size $n=9$ from a population and we observed the following triangular fuzzy data: and using of the ability of package "it MINITAB 13", we show the percentiles and histogram of $t\left(\tilde{\mathbf{x}}_{c}^{*^{b}}\right)$ in the following using 10,000 bootstrap samples. If $B=10,000$, the estimate of the $5 \%$ point is the 500th largest value of the $t\left(\tilde{\mathbf{x}}_{c}^{*^{b}}\right) \mathrm{s}$ and the estimate of the $95 \%$ point is the 9500th largest value of the $t\left(\tilde{\mathbf{x}}_{c}^{*^{b}}\right) s$.

The $\alpha$-cuts of fuzzy bootstrap confidence interval $(\gamma=0.05$ or $90 \%)$ using fuzzy data is

$$
\begin{aligned}
\widetilde{\Pi}_{\alpha}= & \left\{\left[\frac{1}{9} \sum_{i=1}^{9} x_{i}-1.781 \frac{20.36}{\sqrt{9}}, \quad \frac{1}{9} \sum_{i=1}^{9} x_{i}+2.088 \frac{20.36}{\sqrt{9}}\right],\right. \\
& \left.x_{i} \in \tilde{x}_{i \alpha}, i=1,2, \ldots, 9\right\}=[46.153+2.22 \alpha, 76.167-1.556 \alpha] .
\end{aligned}
$$

We show the following the distribution of $t\left(\tilde{\mathbf{x}}_{c}^{*^{b}}\right)$ computed using 10000 bootstrap samples (Fig. 4).

We obtain the $\alpha$-cuts of the so-called fuzzy test statistics

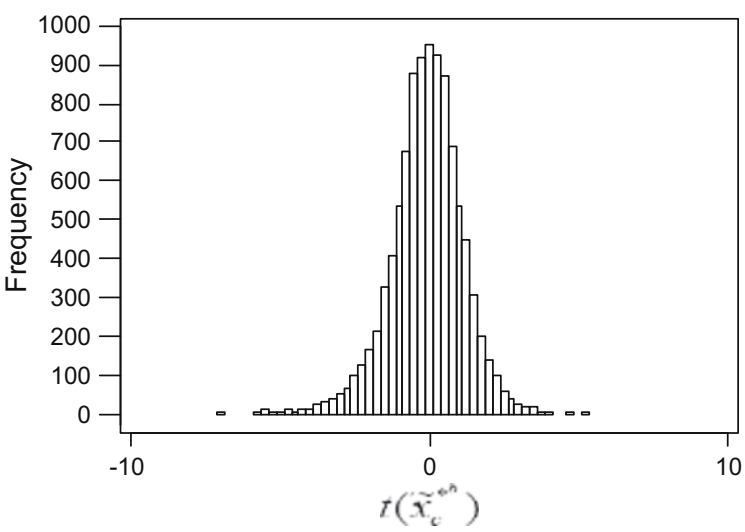

Fig. 4. bootstrap distribution of $t\left(\tilde{\mathbf{x}}_{c}^{*^{b}}\right)$. 
$\widetilde{Z}_{\alpha}=\frac{\widetilde{\Pi}_{\alpha}-\tilde{\theta}_{0 \alpha}}{\frac{s_{(\tilde{\mathbf{x}})}}{\sqrt{n}}}$,

and use the fuzzy test statistics to provide an approach for testing above fuzzy hypotheses, based on the following assumptions (see Fig. 5).

\section{- ASSUMPTIONS}

1. $C_{T}$ be the total area under $\widetilde{Z}$.

2. $C_{1}$ and $C_{2}$ be the areas according to Fig. 5 .

3. $C_{R}=C_{1}+C_{2}$

\section{- DECISION RULE}

- If $\frac{C_{R}}{C_{T}} \leqslant 2 \gamma$, then we accept $H_{0}$.

- If $\frac{C_{R}}{C_{T}} \geqslant 2 \gamma$, then we reject $H_{0}$.

Example 3.2. Consider Table 1. Now suppose that we want to test the following fuzzy hypotheses

$\left\{\begin{array}{l}\widetilde{H}_{0}: \theta \quad \text { is }(57,60,62) \\ \widetilde{H}_{1}: \theta \text { is } \operatorname{not}(57,60,62) .\end{array}\right.$

Here, $\widetilde{H}_{0}$ suggests that $\theta$ is approximation 60 , and $\widetilde{H}_{1}$ suggests that $\theta$ is away from 60 .

We have $\widetilde{Z}_{\alpha}=[-2.339+0.622 \alpha, 2.899-0.671 \alpha], C_{R}=C_{1}+$ $C_{2}=0.061+0.18=0.241, C_{T}=3.39$. since $\frac{C_{R}}{C_{T}}=0.071 \leqslant 0.1$, thus accept $H_{0}$ (Table 2).

3.2. Testing fuzzy simple hypothesis fuzzy against the fuzzy left onesided hypothesis

We want to test the fuzzy null hypotheses

$\left\{H_{0}: \theta\right.$ is approximately $\theta_{0}$,

$\left\{H_{1}: \theta\right.$ is certainly smaller than $\theta_{0}$.

We obtain the $\alpha$-cuts of the so-called fuzzy test statistics

$\widetilde{Z}_{\alpha}=\frac{\widetilde{\Pi}_{\alpha}-\widetilde{\theta}_{0 \alpha}}{\frac{s \widetilde{(\mathbf{x})}}{\sqrt{n}}}$,

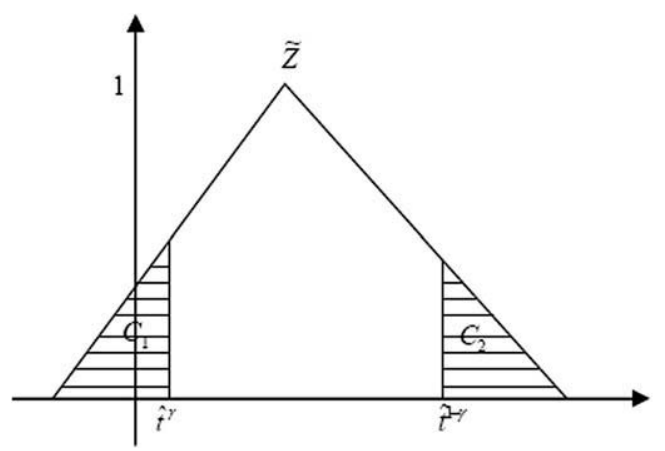

Fig. 5. $\widetilde{Z}$ in testing fuzzy simple hypothesis versus fuzzy two-sided hypothesis.

Table 1

Fuzzy random sample of size $n=9$ from a population.

\begin{tabular}{llllll}
\hline$N$ & Observation & $N$ & Observation & $N$ & Observation \\
\hline 1 & $(32,35,40)$ & 4 & $(60,63,63)$ & 7 & $(70,73,75)$ \\
2 & $(80,82,82)$ & 5 & $(41,45,47)$ & 8 & $(54,56,59)$ \\
3 & $(60,60,60)$ & 6 & $(93,95,96)$ & 9 & $(34,35,36)$ \\
\hline
\end{tabular}

Table 2

Percentiles of the $t$ distribution with 8 degree of freedom, the $N(0,1)$ distribution and the bootstrap distribution of $t\left(\tilde{\mathbf{x}}_{c}^{*^{b}}\right)$.

\begin{tabular}{lllll}
\hline Percentile & $5 \%$ & $10 \%$ & $90 \%$ & $95 \%$ \\
\hline$t_{8}$ & -1.86 & -1.40 & 1.40 & 1.86 \\
Normal & -1.65 & -1.28 & 1.28 & 1.65 \\
Bootstrap & -2.088 & -1.537 & 1.319 & 1.781 \\
\hline
\end{tabular}

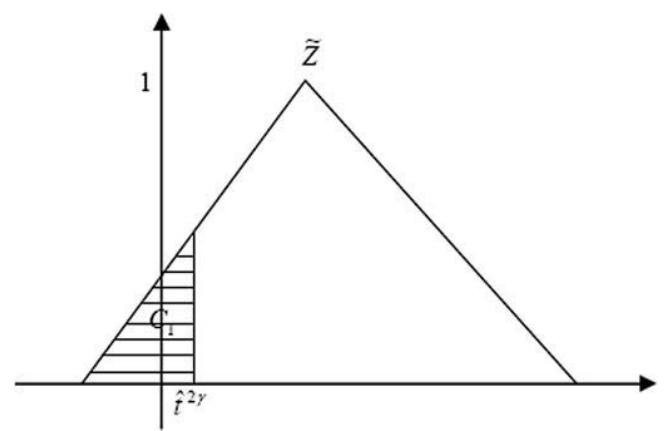

Fig. 6. $\widetilde{Z}$ in testing fuzzy simple hypothesis versus fuzzy left one-sided hypothesis.

and use the fuzzy test statistics to provide an approach for testing above fuzzy hypotheses, based on the following assumptions (see Fig. 6)

\section{- ASSUMPTIONS}

1. $C_{T}$ be the total area under $\widetilde{Z}$.

2. $C_{1}$ be the area according to Fig. 6 .

3. $C_{R}=C_{1}$

\section{- DECISION RULE}

- If $\frac{C_{R}}{C_{T}} \leqslant \gamma$, then we accept $H_{0}$.

- If $\frac{C_{R}^{T}}{C_{T}} \geqslant \gamma$, then we reject $H_{0}$.

Example 3.3. Consider Table 1. Now suppose that we want to test the following fuzzy hypotheses

$\left\{\begin{array}{l}\widetilde{H}_{0}: \theta \quad \text { is approximately }(., 60,62), \\ \widetilde{H}_{1}: \theta \text { is certainly smaller than }(., 60,62) .\end{array}\right.$

We have $\widetilde{Z}_{\alpha}=[-2.339+0.622 \alpha, 2.382-0.23 \alpha], C_{R}=C_{1}=0.061$, $C_{T}=2.79$. since $\frac{C_{R}}{C_{T}}=0.022 \leqslant 0.05$, thus accept $H_{0}$.

3.3. Testing fuzzy simple hypothesis fuzzy against the fuzzy right onesided hypothesis

We want to test the fuzzy null hypotheses

$\left\{\begin{array}{l}H_{0}: \theta \quad \text { is approximately } \theta_{0}, \\ H_{1}: \theta \quad \text { is certainly larger than } \theta_{0} .\end{array}\right.$

We obtain the $\alpha$-cuts of the so-called fuzzy test statistics

$\widetilde{Z}_{\alpha}=\frac{\widetilde{\Pi}_{\alpha}-\widetilde{\theta}_{0 \alpha}}{\frac{s_{(\tilde{x})}}{\sqrt{n}}}$,

and use the fuzzy test statistics to provide an approach for testing above fuzzy hypotheses, based on the following assumptions (see Fig. 7) 


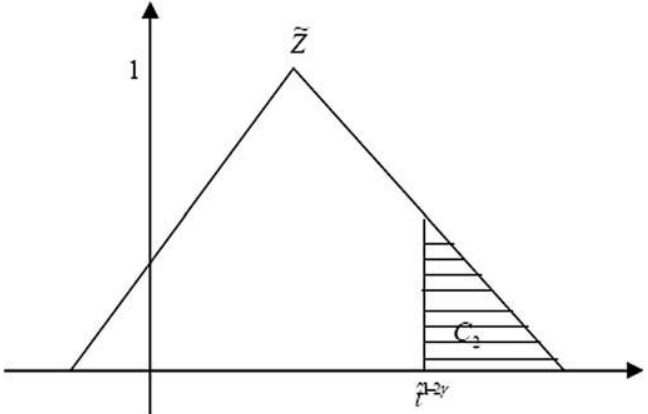

Fig. 7. $\widetilde{Z}$ in testing fuzzy simple hypothesis versus fuzzy left one-sided hypothesis.

\section{- ASSUMPTIONS}

1. $C_{T}$ be the total area under $\widetilde{Z}$.

2. $C_{2}$ be the area according to Fig. 7.

3. $C_{R}=C_{2}$

\section{- DECISION RULE}

- If $\frac{C_{R}}{C_{T}} \leqslant \gamma$, then we accept $H_{0}$.

- If $\frac{C_{R}}{C_{T}} \geqslant \gamma$, then we reject $H_{0}$.

Example 3.4. Consider Table 1. Now suppose that we want to test the following fuzzy hypotheses

$\left\{\widetilde{H}_{0}: \theta\right.$ is approximately $(28,30,$.$) ,$

$\left\{\widetilde{H}_{1}: \theta\right.$ is certainly larger than $(28,30,$.$) .$

We have $\widetilde{Z}_{\alpha}=[2.38+0.327 \alpha, 7.097-0.524 \alpha], C_{R}=C_{2}=2.772$, $C_{T}=16.9$. since $\frac{C_{R}}{C_{T}}=0.164 \geqslant 0.05$, thus reject $H_{0}$.

\section{Bootstrap testing fuzzy hypotheses based on fuzzy test statistic for variance}

Suppose that we have canonical fuzzy random samples $\tilde{\mathbf{x}}=\left(\tilde{x}_{1}, \tilde{x}_{2}, \ldots, \tilde{x}_{n}\right)$. We generate $B$ bootstrap fuzzy random sample $\tilde{\mathbf{X}}^{*^{1}}, \tilde{\mathbf{x}}^{*^{2}}, \ldots, \tilde{\mathbf{x}}^{*^{B}}$ and for each we compute (Fig. 8)

$\chi^{2 *^{b}}=\frac{(n-1) s_{(\tilde{\mathbf{x}})}^{2 *^{b}}}{s_{(\tilde{\mathbf{x}})}^{2}} \quad b=1,2, \ldots, B$

where
(1) $s_{(\widetilde{\mathbf{x}})}^{2 *^{b}}=\frac{1}{n-1} \sum_{i=1}^{n} d^{2}\left(\tilde{x}_{i}^{*^{b}}, \widetilde{\mathbf{x}}^{*}\right)$.
(2) $d$ is Yao-Wu signed distance.
(3) $\widetilde{\mathbf{X}}^{*}=\frac{1}{n} \oplus_{i=1}^{n} \tilde{x}_{i}^{t^{b}}$.
(4) $s_{\widetilde{(\mathbf{x})}}^{2}=\frac{1}{n-1} \sum_{i=1}^{n} d^{2}\left(\tilde{x}_{i}, \widetilde{\mathbf{x}}\right)$.

The $\gamma$ th percentile of $\chi^{2 *^{b}}$ is estimated by the value $\hat{t}^{\gamma}$ such that $\frac{\#\left\{\chi^{2 *^{b}} \leqslant \hat{t}^{\gamma}\right\}}{B}=\gamma$.

Finally, the $\alpha$-cuts of bootstrap confidence interval using fuzzy data is

$\widetilde{\Pi}_{\alpha}^{*}=\left\{\left[\frac{\sum_{i=1}^{n}\left(x_{i}-\overline{\mathbf{x}}\right)^{2}}{\hat{t}^{1-\gamma}}, \quad \frac{\sum_{i=1}^{n}\left(x_{i}-\overline{\mathbf{x}}\right)^{2}}{\hat{t}^{\gamma}}\right]: x_{i} \in \tilde{x}_{i \alpha}, \quad i=1,2, \ldots, n\right\}$,

whenever its membership function is given by

$\mu_{\widetilde{\Pi}^{*}}(y)=\sup _{0 \leqslant \alpha \leqslant 1} \alpha I_{\widetilde{\Pi}_{\alpha}^{*}}(y)$.

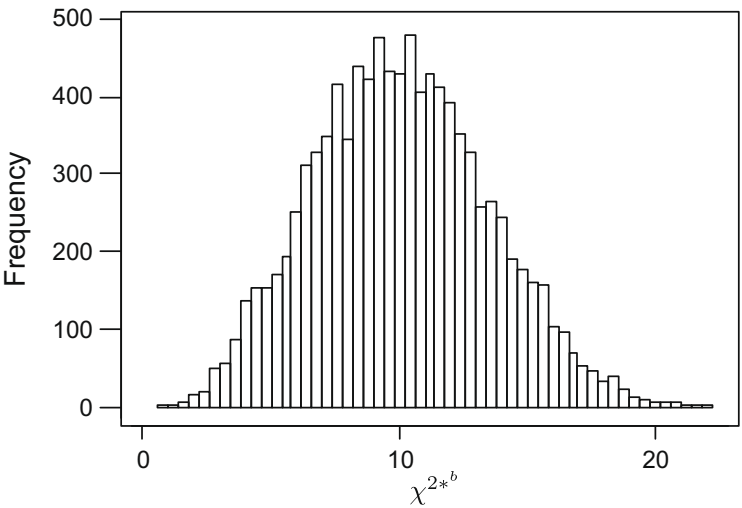

Fig. 8. Bootstrap distribution of $\chi^{2 *^{b}}$.

Table 3

Fuzzy random sample of size $n=12$ from a population.

\begin{tabular}{llllll}
\hline$N$ & Observation & $N$ & Observation & $N$ & Observation \\
\hline 1 & $(33,35,36)$ & 5 & $(60,63,66)$ & 9 & $(100,103,105)$ \\
2 & $(80,82,84)$ & 6 & $(70,70,72)$ & 10 & $(54,56,58)$ \\
3 & $(85,87,87)$ & 7 & $(70,73,76)$ & 11 & $(40,40,42)$ \\
4 & $(90,90,90)$ & 8 & $(65,70,73)$ & 12 & $(94,96,99)$ \\
\hline
\end{tabular}

Example 4.1. Suppose that we have taken a fuzzy random sample of size $n=12$ from a population and we observed the following triangular fuzzy data: (Table 3 ).

The last line of Table 4 shows the percentiles of $\chi^{2 *^{b}}$ for variance computed using 10000 bootstrap samples.

The fuzzy bootstrap confidence interval $(\gamma=0.05$ or $90 \%)$ using fuzzy data is

$\widetilde{\Pi}_{\alpha}^{*}=\left\{\left[\frac{\sum_{i=1}^{12}\left(x_{i}-\overline{\mathbf{x}}\right)^{2}}{15.27}, \quad \frac{\sum_{i=1}^{12}\left(x_{i}-\overline{\mathbf{x}}\right)^{2}}{4.523}\right]: x_{i} \in \tilde{x}_{i \alpha} \quad i=1,2, \ldots, n\right\}$,

and we have for some $\alpha^{\prime}$ s

\begin{tabular}{|c|c|c|c|}
\hline$\alpha$ & 0 & 0.1 & 0.2 \\
\hline $\begin{array}{r}\text { Confidence } \\
\text { interval }\end{array}$ & $\begin{array}{l}{[317.68,} \\
1115.83]\end{array}$ & $\begin{array}{l}{[318.1,} \\
1112.66]\end{array}$ & $\begin{array}{l}\text { [318.39, } \\
1109.59]\end{array}$ \\
\hline$\alpha$ & 0.3 & 0.4 & 0.5 \\
\hline $\begin{array}{r}\text { Confidence } \\
\text { interval }\end{array}$ & $\begin{array}{l}{[318.8,} \\
1106.63]\end{array}$ & $\begin{array}{l}{[319.24,} \\
1103.24]\end{array}$ & $\begin{array}{l}\text { [319.71, } \\
\text { 1101.02] }\end{array}$ \\
\hline$\alpha$ & 0.6 & 0.7 & 0.8 \\
\hline $\begin{array}{r}\text { Confidence } \\
\text { interval }\end{array}$ & $\begin{array}{l}{[320.21,} \\
1098.38]\end{array}$ & $\begin{array}{l}{[320.74,} \\
1095.84]\end{array}$ & $\begin{array}{l}{[321.3,} \\
1093.41]\end{array}$ \\
\hline$\alpha$ & 0.9 & 1 & \\
\hline $\begin{array}{r}\text { Confidence } \\
\text { interval }\end{array}$ & $\begin{array}{l}{[321.9,} \\
1091.08]\end{array}$ & $\begin{array}{l}{[322.52,} \\
1088.86]\end{array}$ & \\
\hline
\end{tabular}

We obtain the $\alpha$-cuts of the so-called fuzzy test statistics $\widetilde{Z}_{\alpha}^{*}=\frac{(n-1) \widetilde{\Pi}_{\alpha}^{*}}{\widetilde{\theta}_{0 \alpha}}$,

and now we can similarly apply the previous section for analyzing of this section. 
Table 4

Percentiles of the $\chi^{2}$ distribution with 7 and 11 degrees of freedom and the bootstrap distribution of $\chi^{2 *^{b}}$.

\begin{tabular}{llllllll}
\hline Percentile & 0.005 & 0.01 & 0.025 & 0.05 & 0.95 & 0.975 & 0.99 \\
\hline$\chi^{2,7}$ & 0.989 & 1.239 & 1.69 & 2.167 & 14.067 & 16.013 & 20.99 \\
$\chi^{2,11}$ & 2.603 & 3.053 & 3.816 & 4.575 & 19.675 & 21.92 \\
Bootstrap & 2.699 & 3.077 & 3.85 & 4.523 & 15.27 & 24.725 \\
\hline
\end{tabular}

\section{Conclusion}

The proposed procedure is based on a significance level. Extension of the proposed method to test the parameters of linear models, such as regression models, design of experiment is a potential area for the future work. We can use the Yao-Wu signed distance for estimation of coefficients of linear models and apply the proposed method in this paper.

\section{Acknowledgements}

Authors are grateful to the referees of the journal for their suggestions and would like to thank the Co-Editor-in-Chief Doctor Vijayakumar for the helpful comments and useful helps.

\section{References}

Arnold, B. F. (1996). An approach to fuzzy hypothesis testing. Metrika, 44, 119-126. Arnold, B. F. (1998). Testing fuzzy hypothesis with crisp data. Fuzzy Sets and Systems, $94,323-333$

Buckley, J. J. (2005). Fuzzy probabilities: New approach and applications. Berlin, Heidelberg: Springer-Verlag.

Buckley, J. J. (2006). Fuzzy probability and statistics. Berlin, Heidelberg: SpringerVerlag.
Cheng, C. (1998). A new approach for ranking fuzzy numbers by distance method. Fuzzy Sets and Systems, 95, 307-317.

Desimpelaere, C., \& Marchant, T. (2007). An empirical test of some measurementtheoretic axioms for fuzzy sets. Fuzzy Sets and Systems, 158, 1348-1359.

Filzmoser, P., \& Viertl, R. (2004). Testing hypotheses with fuzzy data: The fuzzy pvalue. Metrika, 59, 21-29.

Gonzalez-Rodriguez, G., Montenegro, M., Colubi, A., \& Gil, M. A. (2006). Bootstrap techniques and fuzzy random variables: Synergy in hypothesis testing with fuzzy data. Fuzzy Sets and Systems, 157, 2608-2613.

Hyniewicz, O. (2006). Possibilitic decisions and fuzzy statistical tests. Fuzzy Sets and Systems, 157, 2665-2673.

Korner, R. (2000). An asymptotic $\alpha$-cut for the expectation of random fuzzy variables. Journal of Statistical Planning and Inferences, 83, 331-346.

Modarres, M., \& Sadi-Nezhad, S. (2001). Ranking fuzzy numbers by preference ratio. Fuzzy Sets and Systems, 118, 429-436.

Montenegro, M., Colubi, A., Casals, M. R., \& Gil, M. A. (2004). Asymptotic and bootstrap techniques for testing the expected value of a fuzzy random variable. Metrika, 59, 31-49.

Nojavan, M., \& Ghazanfari, M. (2006). A fuzzy ranking method by desirability index. Journal of Intelligent and Fuzzy Systems, 17, 27-34

Taheri, S. M., \& Behboodian, J. (1991). Neyman-Pearson Lemma for fuzzy hypotheses testing. Metrika, 49, 3-17.

Thompson, E. A., \& Geyer, C. J. (2007). Fuzzy p-values in latent variable problems. Biometrika, 94, 49-60.

Torabi, H., Behboodian, J., \& Taheri, S. M. (2006). Neyman-Pearson Lemma for fuzzy hypotheses testing withy vague data. Metrika, 64, 289-304.

Viertl, R. (2006). Univariate statistical analysis with fuzzy data. Computational Statistics and Data Analysis, 51, 133-147.

Yao, J. S., \& Wu, K. (2000). Ranking fuzzy numbers based on decomposition principle and signed distance. Fuzzy Sets and Systems, 11, 275-288. 